\title{
CAMPANHA DE DOAÇÃO DE ÓRGÃOS: UM RELATO DE EXPERIÊNCIA
}

\author{
Organ donation campaign: an experience report
}

\author{
Samantha Sartore Duque Estrada Medeiros, Fernanda de Brito Fortuna, Gabriela Acurcio Barbosa, \\ Maria Isabel Barêa Fávero Reis, Beatriz de Oliveira Onório, Beatriz Carvalho Borges, \\ Beatriz Ventura Evangelista, Pâmella Hellen Sousa Martins, Bárbara Zantut Wittmann, \\ Rafaella Gusman Lorencetti, Ana Luisa Costa Bezerra
}

\section{RESUMO}

Introdução: A doação de órgãos representa enorme potencial de salvar inúmeras vidas, porém, 40\% dos familiares de falecidos por morte encefálica ainda negam a doação. Isso ocorre devido ao processo de luto e à falta de esclarecimento sobre o tema, impactando negativamente o número de doadores e ampliando as filas de espera. É, portanto, essencial conscientizar a população quanto à importância da doação de órgãos. Objetivo: Relatar a experiência da Campanha de Doação de Órgãos como método inovador e transformador importante à conscientização da população e ao incentivo à doação de órgãos. Métodos: Trata-se de estudo descritivo, que objetiva relatar a experiência de acadêmicos do curso de graduação de medicina da universidade PUC-Campinas, durante a organização e participação da Campanha de Doação de Órgãos. Resultados: O Comitê PUC-Campinas da Federação Internacional da Associação de Estudantes de Medicina do Brasil (IFMSA Brazil), em parceria com a Organização de Procura de Órgãos e o Parque Dom Pedro Shopping realizaram, em 19 de maio de 2018, uma campanha na praça de alimentação do Shopping em Campinas. Foi oferecida capacitação prévia aos voluntários por meio de depoimentos e palestra multidisciplinar. O público foi abordado individualmente e de forma dinâmica, através da entrega de fichas que simulavam o número de expectantes em filas de transplante no Brasil. Desse modo, buscou-se induzir a curiosidade sobre o assunto, esclarecer dúvidas e informar quanto à possibilidade de se declarar doador de órgãos, estimulando o diálogo com familiares a respeito do tema. Como feedback, o público informou acerca de conversa prévia sobre ser doador e do desejo de sê-lo, após o evento. Foram abordadas 422 pessoas, sendo que pouco mais de um terço $(35,3 \%)$ destas nunca havia discutido sobre doação de órgãos e, após o evento, quase a totalidade $(94,5 \%)$ demonstrou desejo de ser doador. Dentre 25 voluntários, nenhum havia participado de atividade sobre o tema e 24 consideraram-se aptos a orientar terceiros, após a capacitação. Conclusão: A Campanha atuou sobre grande público e fomentou a conscientização de acadêmicos e dos participantes, e o incentivo ao diálogo, a fim de evitar que limitantes preveníveis como a falta de informação ou compreensão do tema impeçam o avanço das doações de órgãos, de significativa demanda.

Descritores: Obtenção de Tecidos e Órgãos; Sensibilização Pública; Comunicação; Família.

Instituição:

Faculdade de Medicina da Pontifícia Universidade Católica de Campinas - Campinas, São Paulo, Brasil

\section{Correspondência:}

Samantha Sartore Duque Estrada Medeiros

Rua Tupiniquins, $n^{\circ} 104$, Unidade 45. CEP 13.335-540, Indaiatuba/SP (19) 99736-5073

samanthasdem@terra.com.br

Recebido em: 01/10/2019

Aceito em: 31/10/2019

\section{INTRODUÇÃO}

Diferentemente de outras terapêuticas médicas, o transplante de órgãos é realizado apenas através da doação devidamente autorizada por familiares de potenciais doadores. ${ }^{1}$ Doar órgãos é um ato de amor e solidariedade ao próximo, porém, por depender de decisão tomada durante um momento de luto, por vezes associada à falta de esclarecimentos sobre o tema, muitos dos familiares sentem-se apreensivos e indecisos em autorizar a doação. ${ }^{2}$ 
Tal fato pode impactar negativamente o número de possíveis doadores e, consequentemente, aumentar a demora nas filas de espera, o que para alguns é questão de vida ou morte. ${ }^{2}$

Segundo dados da Associação Brasileira de Transplante de Órgãos (ABTO), o número de transplante de órgãos no Brasil aumentou $14 \%$ em $2017 .{ }^{3}$ Com essa estatística, o país destaca-se positivamente no contexto mundial. Ressalta-se ainda o potencial de um único doador, que pode salvar ou ao menos melhorar a qualidade de vida de mais de vinte pessoas. ${ }^{4}$ No entanto, o índice de transplante de órgãos no país ainda se mostra insuficiente para suprir a demanda nacional. ${ }^{4}$

Dentre os fatores limitantes que incluem uma série de variáveis, desde a qualidade da assistência pública nas urgências até a autorização da doação, a negativa familiar ainda se ressalta no Brasil. ${ }^{5}$ Nesse cenário, torna-se evidente a importância da participação ativa da população para influir de forma positiva nas estatísticas de doações de órgãos, em âmbito nacional, também incluindo a participação essencial dos profissionais de saúde, os quais têm a responsabilidade de se informar sobre o processo de doação de órgãos, para que possam assegurar orientações e responder às possíveis dúvidas sobre o assunto, de modo satisfatório. ${ }^{6}$

Essas limitações projetam-se também no meio acadêmico da Medicina. Segundo estudo realizado com 347 estudantes de medicina, 70\% declararam ter conhecimento regular ou péssimo do assunto, o que pode contribuir para perpetuar a falta de conhecimento, reduzindo a propagação de informações que fomentem e incentivem a doação. ${ }^{7}$

Diversas campanhas de incentivo e desmistificação da doação de órgãos visam aumentar as taxas de consentimento para órgãos transplantáveis, fato considerado uma estratégia promissora para amenizar a lacuna entre a necessidade e oferta de órgãos. ${ }^{8}$ No Brasil, a principal delas é organizada pelo Ministério da Saúde, especialmente relacionada à criação do "Setembro Verde". ${ }^{9}$ Existem também esforços da própria ABTO em apoiar campanhas de incentivo à doação de órgãos e tecidos, em diversas regiões do país, ${ }^{10}$ incluindo o estado de São Paulo. Apesar das diferentes abordagens, todas compartilham do objetivo central de orientar, tanto a população geral quanto os profissionais de saúde, em prol do estímulo à doação.

Motivados a contribuir para a conscientização do público em geral e a difusão do significado humanitário, científico e moral da doação de órgãos e tecidos para transplante, a IFMSA Brazil Comitê PUC-Campinas estruturou a Campanha de Doação de Órgãos.

\section{MÉTODOS}

Trata-se de um estudo descritivo, que objetiva relatar a experiência de acadêmicos do curso de graduação de Medicina, da Pontifícia Universidade Católica de Campinas, filiados ao comitê local PUC-Campinas da IFMSA Brazil, durante a organização e participação da Campanha de Doação de Órgãos, realizada em 19 de maio de 2018, no estabelecimento Parque Dom Pedro Shopping, em Campinas, São Paulo.

\section{RESULTADOS}

A Campanha de Doação de Órgãos foi idealizada e planejada por dez membros do comitê PUC-Campinas da IFMSA Brazil e, como tal, seguiu a missão dessa federação: gerar impacto positivo na sociedade, através da organização de ações por estudantes de Medicina. Para isso, o evento contou com a participação de 25 acadêmicos voluntários, mobilizados por meio da divulgação da campanha, em mídias sociais, como o WhatsApp, Instagram e Facebook, além da exposição de cartazes e banners em locais de grande visibilidade do Campus II da PUC-Campinas.

A campanha foi autossustentável e sem fins lucrativos; o valor simbólico arrecadado com as inscrições dos voluntários foi direcionado para suprir os gastos de transporte e o material de apoio utilizado no dia da ação. Contou ainda com o patrocínio do Shopping Dom Pedro, que ofereceu o devido apoio e a autorização para a realização do evento no estabelecimento, fornecendo também camisetas personalizadas para identificação dos voluntários envolvidos, e da Organização de Procura de Órgãos (OPO) do Hospital de Clínicas da Universidade Estadual de Campinas (UNICAMP), que contribuiu com aula teórica preparatória aos acadêmicos.

Tal capacitação ocorreu na véspera da campanha, no campus II da universidade. A temática de doação de órgãos foi abordada mediante depoimentos de doador e receptor de órgãos e palestra multidisciplinar, ministrada por uma representante da OPO da UNICAMP. A capacitação permitiu: elucidar a importância da doação de órgãos e suas implicações para a sociedade, qualificar os voluntários a orientar familiares de possíveis doadores sobre o processo de doação e esclarecer dúvidas acerca do tema, desconstruir mitos e preconceitos associados, apresentar a complexa logística do processo de transplante de órgãos e tecidos e sua estrutura operacional, além de introduzir o conceito e diagnóstico de morte encefálica. Ao final, os acadêmicos receberam um folder contendo as principais informações discutidas para auxiliá-los no evento. 
Samantha Sartore Duque Estrada Medeiros, Fernanda de Brito Fortuna, Gabriela Acurcio Barbosa, Maria Isabel Barêa Fávero Reis, Beatriz de Oliveira Onório, Beatriz Carvalho Borges, Beatriz Ventura Evangelista, Pâmella Hellen Sousa Martins, Bárbara Zantut Wittmann, Rafaella Gusman Lorencetti, Ana Luisa Costa Bezerra

Em sequência, a Campanha de Doação de Órgãos ocorreu na praça de alimentação do Parque Dom Pedro Shopping, durante o horário de almoço, com duração aproximada de três horas. Estruturou-se um estande de apoio no local com material de divulgação da campanha. Cada voluntário recebeu fichas personalizadas, que simulavam o número de pessoas nas filas de espera para transplante no Brasil, a fim de serem utilizadas como abordagem inicial ao público.

Os clientes do shopping foram abordados de forma randomizada, individual, dinâmica e informal. Uma vez com a atenção e a curiosidade sobre o assunto instituídas na maioria das pessoas, os voluntários esclareceram dúvidas e informaram quanto à possibilidade de se declararem doadores de órgãos, estimulando também a conversa a respeito do tema entre o público alvo e seus familiares.

Com o intuito acadêmico de mensurar o impacto do evento, foi distribuído um breve questionário, preenchido voluntariamente pelos indivíduos abordados durante a campanha; outro formulário foi entregue aos voluntários para avaliar a campanha e a capacitação prévia. A avaliação de impacto é uma ferramenta importante no meio acadêmico; é especialmente utilizada em ações da IFMSA Brazil para identificar a efetividade ou o impacto positivo de determinada ação, sob a perspectiva do público alvo, bem como fatores de melhora e sugestões para aprimoramentos futuros.

A Campanha obteve amplo alcance, conscientizando 422 pessoas, em três horas. Os voluntários constataram que pouco mais de um terço $(35,3 \%)$ dos abordados nunca havia discutido sobre doação de órgãos e, após o evento, quase a totalidade $(94,5 \%)$ demonstrou desejo de ser doador, alegando que compartilharia esse interesse com os familiares. Ainda, foi percebido que a existência de comunicação prévia com familiares mostrou-se mais elevada conforme maior a idade dos indivíduos. Tal fato, aliado à maior parte dos abordados no evento ser composta por adultos jovens, foi identificado pelos voluntários como outro ponto favorável da campanha, por permitir a mobilização precoce de um grupo considerável de potenciais doadores.

\section{DISCUSSÃO}

Pôde-se perceber que o principal objetivo de incentivar a comunicação familiar acerca da doação de órgãos foi atingido, tendo havido efetiva mobilização dos indivíduos à doação, através de abordagem personalizada e informal, que compreendeu uma comunicação harmoniosa e de grande êxito, capaz de atrair o público pela curiosidade e interesse, tendo sido favorável à transmissão de conhecimento e, consequentemente, ao propósito da campanha.
Tal característica é ressaltada, pois um dos principais desafios envolvidos na orientação acerca da doação de órgãos é, justamente, o estabelecimento de um contato que promova a escuta ativa do público, por ser um assunto considerado complexo e relacionado a crenças e à desinformação. Na prática, a comunicação interpessoal é sugerida como um diferencial positivo quando comparada às abordagens restritas à mídia e pode ser um dos principais contribuintes para que as diversas campanhas de conscientização sobre a doação de órgãos sejam uma estratégia interessante para promover a redução nas taxas de recusa familiar. Tal pressuposto parte de uma metanálise que englobou vinte e três campanhas de educação pública em doação de órgãos realizadas nos Estados Unidos e mais de quarenta e três mil entrevistados. Nesse estudo, foi observado, após as campanhas, um aumento de $5 \%$ de ganho associado à doação de órgãos, o que inclui a manifestação do desejo de doar órgãos, a comunicação a familiares e a assinatura de cartão doador. ${ }^{8,11}$

Além disso, há maior tendência de consentimento dos familiares quando bem orientados sobre o conceito de morte encefálica e a finalidade humanística da doação. ${ }^{5}$ De fato, Simpkin et al. (2009) revisaram os principais fatores modificáveis influenciadores na decisão de familiares em permitir a doação de órgãos e constataram que o maior fator estudado é a abordagem e experiência do responsável pela solicitação, podendo potencializar a resposta afirmativa, se realizada adequadamente. ${ }^{12}$ Nesse sentido, a campanha propiciou fatores fundamentais para o sucesso na captação de órgãos: não só maior conhecimento ao público, mas também o desenvolvimento prático de habilidades comunicativas do futuro profissional de saúde.

Ademais, a faixa etária jovem, majoritariamente abordada na campanha, mostrou-se um potencial promissor do evento. Cabe destacar que, em 2017, a faixa etária com maior número de doadores no Brasil (32\%) foi entre 50 e 64 anos, seguida pela faixa de 35 a 49 e 18 a 34 anos, com $29 \%$ e $21 \%$ respectivamente. ${ }^{3}$ Estimular precocemente a conversa familiar busca ajudar no processo de decisão, dificultado pela condição de estresse que as famílias vivenciam com a perda do familiar. ${ }^{5}$

Assim, a Campanha conseguiu esclarecer dúvidas e estimular a conversa familiar de maneira efetiva, a fim de combater o principal entrave na efetivação de transplante de órgãos já descrito na literatura: a negação da família.,12 Visando modificar essa realidade e promover aumento do número de doadores, a Campanha de Doação de Órgãos engajou-se em contribuir para a diminuição do número de recusas familiares a nível local. A capacitação dos voluntários foi especialmente impactante no desenvolvimento e 
aprendizado dos acadêmicos, que evoluíram da falta de informação à capacidade e segurança em educar terceiros: nenhum dos trinta e cinco voluntários havia participado de alguma atividade sobre o tema e vinte e quatro deles consideraram-se aptos a orientar terceiros, após a capacitação.

O evento como um todo permitiu instruir e qualificar os estudantes, contribuindo para amenizar a lacuna da temática na graduação e possibilitando-os a se tornarem capazes de explanar com propriedade sobre o processo de doação de órgãos e evitar a recusa familiar, tornando-os futuros profissionais de saúde mais preparados e protagonistas em prol desta causa.

\section{CONCLUSÃO}

A elevada demanda nacional da doação de órgãos associada à falta de informação acerca do tema revela a necessidade de campanhas de intervenção, com intuito de conscientizar a população e incentivar o diálogo familiar. A Campanha de Doação de Órgãos, estruturada em formato de capacitação e campanha, demonstrou ser uma ação local com capacidade transformadora e de grande impacto, tanto para os acadêmicos quanto para o público alvo, conseguindo atingir seus objetivos com maestria e resultando em uma repercussão maior do que a esperada. A abordagem promoveu a capacitação dos futuros profissionais de saúde para se tornarem agentes transformadores do atual cenário, bem como promoveu a confiança do público e o desejo de realizar doação de órgãos, possibilitando, através de futuros diálogos com familiares e amigos, a propagação de tal intervenção em progressão geométrica. Espera-se que a publicação científica, como ferramenta de difusão e democratização do conhecimento, permita a expansão dessa ação de nível local para esferas mais abrangentes, incluindo a possível união de instituições, em escala regional e nacional, a fim de tornar ainda mais efetivo o impacto positivo sobre a doação de órgãos no Brasil.

\section{ABSTRACT}

Introduction: Organ donation represents an enormous potential to save numerous lives, but $43 \%$ of relatives of brain-dead patients still refuse donating. This is due to the grieving process and the lack of elucidation on the subject, which negatively impact on the number of donors and increases the waiting lines. Therefore, it is essential to raise awareness among the population on the importance of organ donation. Purpose: To report the experience of the Organ Donation Campaign as an important innovating and transforming method to raise awareness among the population and encourage organ donation. Methods: This is a descriptive study aiming to report the experience of undergraduate medical students of PUC-Campinas University during organization and participation of Organ Donation Campaign. Results: The PUC-Campinas Committee of International Federation of Medical Students Association of Brazil (IFMSA Brazil), in partnership with Organ Procurement Organization and Parque Dom Pedro Shopping organized on May 19, 2018, the campaign in the food court of the Shopping Center in Campinas. Medical students who volunteered to participate were offered training through testimonials and multidisciplinary lecture. The public was approached individually and dynamically through by delivering records simulating the number of patients on the Brazilian Transplant Waiting List. Thus, we attempted to induce curiosity about the subject, clarify doubts and inform about the possibility of declaring themselves organ donors by encouraging dialogue with family members on the topic. As feedback, the public reported whether they had already talked about the issue with their families and whether the campaign had aroused their desire to become organ donors. Of 422 people approached, just over a third $(35.3 \%)$ had never discussed organ donation, and after the event, almost all (94.5\%) expressed desire to be a donor. Among 25 volunteers, none had participated in activities on the subject and 24 considered themselves able to guide others after the training. Conclusion: The Campaign promoted awareness among the general public and academics, besides of encouraging the dialogue in order to avoid that preventable constraints such as the lack of information or comprehension of the subject to hinder the advance of organ donations, which have a significant demand.

Keywords: Tissue and Organ Procurement; Public Awareness; Communication; Family. 
Samantha Sartore Duque Estrada Medeiros, Fernanda de Brito Fortuna, Gabriela Acurcio Barbosa, Maria Isabel Barêa Fávero Reis, Beatriz de Oliveira Onório, Beatriz Carvalho Borges, Beatriz Ventura Evangelista, Pâmella Hellen Sousa Martins, Bárbara Zantut Wittmann, Rafaella Gusman Lorencetti, Ana Luisa Costa Bezerra

\section{REFERÊNCIAS}

1) ASSOCIAÇÃO BRASILEIRA DE TRANSPLANTE DE ÓRGÃOS, Manual: Entenda a doação de órgãos, decidase pela vida. [Internet] 2002 Ago [cited 2018 May 07]. Available from: URL: http://www.abto.org.br/abtov03/ Upload/file/entendadoacao.pdf

2) Morais TR. Doação de órgãos: é preciso educar para avançar. Saúde debate. [Internet] 2012 Dec [cited 2018 May 06]; 36(95):633-39 Available from: URL: http://www.scielo.br/scielo.php?script=sci_arttext\&pid =S0103-11042012000400015

3) ASSOCIAÇÃO BRASILEIRA DE TRANSPLANTE DE ÓRGÃOS, Registro Brasileiro de Transplante: dimensionamento dos transplantes no Brasil em cada estado no período Janeiro/Dezembro - 2017. São Paulo: ABTO, 2017. Available from: URL: http://www.abto.org. br/abtov03/Upload/file/RBT/2017/rbt-imprensa-leituracompressed.pdf

4) ASSOCIAÇÃO BRASILEIRA DE TRANSPLANTE DE ÓRGÃOS. Informe Publicitário: Orientações sobre Doação de Órgãos. Available from: URL: http://www.abto.org.br/ estendaamao/files/0_abto_casada_alta.pdf

5) Sadala MLA. A experiência de doar órgãos na visão de familiares de doadores. J. Bras. Nefrol. [Internet]. 2001 Oct [cited 2018 May 06]; 23(3):143-51. Available from: URL: http://bjn.org.br/details/493/pt-BR/a-experiencia-de-doarorgaos-na-visao-de-familiares-de-doadores.

6) Morais TR, Morais MR. Doação de órgãos: é preciso educar para avançar. Saúde em Debate [Internet]. 2012 Dec; 36(95):633-9. Available from: https://doi.org/10.1590/ S0103-11042012000400015.
7) Galvao FHF, Caires RA, Azevedo-Neto RS, Mory EK, Figueira ERR, Otsuzi TS, et al. Conhecimento e opinião de estudantes de medicina sobre doação e transplante de órgãos. [Internet]. [cited 2018 May 06]. Rev. Assoc. Med. Bras. 2007Oct;53(5):401-6. Available from: http:// dx.doi.org/10.1590/S0104-42302007000500015

8) Feeley TH, Moon S-I. Promoting Organ Donation through Public Education Campaigns: A RandomEffects Meta-Analysis. Available from: https://www. organdonor.gov/sites/default/files/about-dot/files/ metaanalysisfinalmanuscript.pdf

9) MINISTÉRIO DA SAÚDE. \#AVIDACONTINUA: DOE ÓRGÃOS. CONVERSE COM SUA FAMÍLIA [Internet]. Available from: https://portalarquivos.saude.gov.br/ campanhas/doeorgaos/downloads/MS_Doacao_ Medico_175x270.pdf

10) BOLETIM INFORMATIVO DA ASSOCIAÇÃO BRASILEIRA DE TRANSPLANTE DE ÓRGÃOS: Edição Especial "VI Campanha de Doação de Órgãos e Tecidos". Julho/Setembro - 2004. São Paulo: ABTO, 2004. Available from: www.transplantes.go.gov.br

11) Aykas A, Uslu A, Şimşek C. Mass media, online social network, and organ donation: Old mistakes and new perspectives. Transplant Proc. 2015;47(4):1070-2.

12) Simpkin AL, Robertson LC, Barber VS, Young JD. Modifiable factors influencing relatives' decision to offer organ donation: Systematic review. BMJ. 2009;338(7702):1061-3.

\section{AGRADECIMENTOS}

Agradecemos a Associação Brasileira de Transplante de Órgãos, pela oportunidade de apresentação de poster no XVI Congresso Brasileiro de Transplantes e pelo convite de publicação do trabalho no Jornal Brasileiro de Transplantes.

À International Federation of Medical Students Association of Brazil, em especial ao comitê PUCCampinas agradecemos pelo incentivo e suporte contínuo na organização do evento.

Por fim, agradecemos a parceria da Organização de Procura de Órgãos - UNICAMP e do Parque Dom Pedro Shopping pelo apoio logístico essencial para efetivação da Campanha de Doação de Órgãos. 